\title{
Cryptococcal Meningitis in Immunocompetent Patient-Case Report
}

\author{
${ }^{1}$ Yanes Brum Bello, ${ }^{1}$ Hanna Guapyassu Machado, ${ }^{1}$ Juliana Freitas Silveira, \\ ${ }^{1}$ Fernanda Schettini, ${ }^{1}$ Gilberto Martins Junior, ${ }^{1}$ Sergio Dortas Junior, \\ ${ }^{1}$ Carlos Henrique Reis, ${ }^{2}$ Marco Orsini, ${ }^{2}$ Peter Salem, ${ }^{3}$ Dionis Machado, \\ ${ }^{3}$ Victor Hugo Bastos, ${ }^{4}$ Amanda Julia Ramos Bezerra, \\ ${ }^{1}$ Fernanda Martins Coelho Catharino and ${ }^{1}$ Antonio Marcos da Silva Catharino \\ ${ }^{1}$ Department of Neurology, \\ General Hospital of Nova Iguaçu, Nova Iguaçu-Rio de Janeiro, Brazil \\ ${ }^{2}$ Department of Neurology, \\ Antonio Pedro University Hospital-Federal Fluminense University-UFF, Niterói-Rio de Janeiro, Brazil \\ ${ }^{3}$ Departament of Physical Therapy, \\ Brain Mapping Lab and Functionality-Federal University of Piauí (UFPI), Brazil \\ ${ }^{4}$ Brain Mapping Lab and Functionallity (UFPI), Brazil
}

Received 2012-05-15, Revised 2012-08-21; Accepted 2013-05-02

\begin{abstract}
Cryptococcal Meningitis (CM) is a rare infection in immunocompetent patients. A kind of central nervous system infection caused by encapsulated yeast-like fungus Cryptococcus neoformans. A 59-year-old man presented to the Neurology Department of Nova Iguacu General Hospital, complaining has felt "muddled" recently and feeling diaphragmatic spasm without any apparent cause. In addition, at neurological examination, the patient was slightly confused and during the mini-mental state examination he scored less than 20 points, feeling "slowed down", no cranial nerve dysfunction, "rigidity of gait as well as of hand movements, more pronounced on the right one, pyramidal signs bilaterally were more intensely noted on the left". His MRI, lumbar puncture, fungal isolation and Nakin Ink were positive to Cryptococcosis while, in turn, HIV tests I and II were both negative. The treatment was started with Amphotericin B 50 mg IV, once a day, plus Dexamethashone. From our clinical case, we decided to do a brief review about Cryptococcoal Meningitis in immunocompetents and Cryptococcoma, researching at MedLine and Pubmed, using terms "Cryptococcal meningitis", "Cryptococcal meningitis in immunocompetent" and "Cryptococcomas". It is concluded that CM in immunocompetents is uncommon, but an important cause of non-acute meningitis, that should be included in the range of causes of preventable blindness. In this sense, this article purposes advertise clinicians and specialists, to recognize the clinical manifestation and diagnosis of cryptococcal meningitis in immunocompetents, trying to avoid a later diagnosis and the following complications.
\end{abstract}

Keywords: Immunocompetent Patient, Neuroinfection, Cryptococcal Meningitis

\section{INTRODUCTION}

Cryptococcal Meningitis (CM) is a serious subacute encapsulated yeast-like fungus, Cryptococcus neoformans. The initial infection site is the lungs which may disseminate hematogenously to the brain and or chronic central nervous system infection caused by meninges (Baddley et al., 2008). Cryptococcus may also

Corresponding Author: Yanes Brum Bello, Department of Neurology, General Hospital of Nova Iguaçu, Nova Iguaçu-Rio de Janeiro-Brazil 
cause symptomatic disease in other organ systems, including the central nervous system, skin, prostate and eyes. Central-nervous-system involvement often manifests itself as meningitis or meningoencephalitis, but may, in some cases, presents itself as isolated cryptococcomas (Goldman et al., 2010). There are two varieties of $C$. neoformans, namely "neoformans variety" and "gatti variety". The $C$. neoformans variety, found in aged pigeon droppings, causes disease in immunocompromised patients including AIDS patients. $C$. neoformans variety gatti is not associated with birds, but grows in the litter around certain species of eucalyptus trees causing disease in immunocompetent hosts, although a study in India has found that the hypothesis of global spread of $C$. gattii through export of Australian Eucalyptus infected seeds is rebutted (Chowdhary et al., 2012). The yeast grows well at $37^{\circ} \mathrm{C}$, its virulence is given in part to its mucopolysaccharide capsule, which deters phagocytosis. While $C$. neoformans has a worldwide distribution, $C$. gattii was more likely to be predominantly located in tropical and subtropical regions, but a recent outbreak in Vancouver Island, British Columbia, suggested this organism has a wider distribution including the Pacific Northwest and Northern California (Goldman et al., 2010). There is a case report in a immunocompetent patient diagnosed at HGNI-Brazil.

\subsection{Case Report}

A 59-year-old man has felt "muddled" recently, showing diaphragmatic spasm without any apparent cause. At neurological examination, the patient was slightly confused and in mini-mental state examination he scored less than 20 points, feeling "slowed down", no cranial nerve dysfunction, "rigidity of gait as well as of hand movements, more pronounced on the right side, pyramidal signs bilaterally more intensely noted on the left". An MRI scan (Fig. 1) of his head showed "nodules with hypo-intensity on T1 and hiper-intensity on T2, consistent with fatty saturation, with signs of heterogeneity inside, showing a subtle post contrast enhancement, in basal ganglia suggesting toxoplasma or cryptococcal infection. A latex agglutination serum test for the cryptococcal antigen was positive (at 1/1200), instead of this, HIV test I e II were both negative. A lumbar puncture was performed. Nankin dye indicated Cryptococcosis and the fungal isolation showed the growth of Cryptococcus neoformans. The treatment started with Amphotericin B $50 \mathrm{mg} \mathrm{IV}$, once a day, plus Dexamethashone.

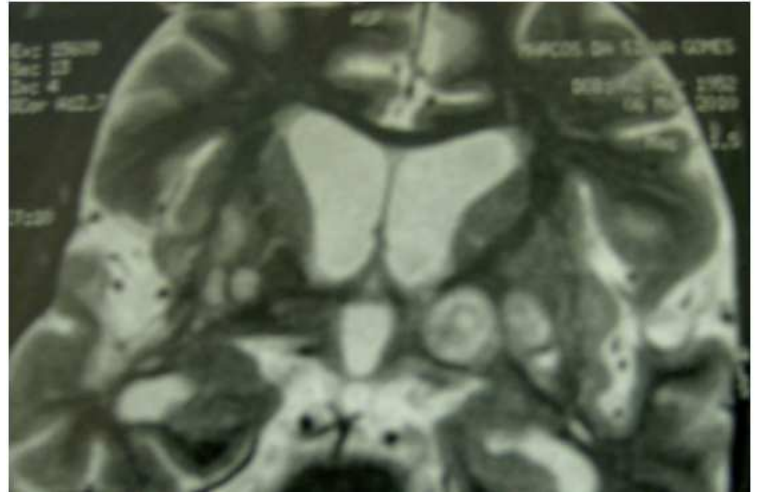

Fig. 1. Image hypointense on $\mathrm{T} 1$, hyperintense on $\mathrm{T} 2$, with limited difusion, capturing contrast in deep gray matter, thalamus regions/capsular beyond the cerebral peduncle, paravermiama cerebellar subcortical white matter and cerebral cortex. Supratentorial ventricular dilatation

\section{MATERIALS AND METHODS}

From our clinical case, we decided to address a brief review about $\mathrm{CM}$ in immunocompetents and Cryptococcoma, researching at Pubmed, using terms "Cryptococcal meningitis", "Cryptococcal meningitis in immunocompetent" and "Cryptococcomas".

\section{RESULTS}

121 articles were found with term "Cryptococcoal Meningitis in immunocompetents", 2155 with "Cryptococcoal Meningitis" and 47 with term "Cryptococcomas". Were selected those with free full text available, excluded those presenting clinical manifestation outside the central nervous system and preferred those about $\mathrm{CM}$ in immunocompetent.

\section{DISCUSSION}

Cryptococcosis is more common in immunocompromised patients, including those with impaired cell-mediated immunity, as in HIV, hematologic malignancies and solid-organ transplant recipients and patients on chronic corticosteroids or other immunosuppressive therapy (Goldman et al., 2010). Other immunocompromised conditions associated to a typical presentation include a alcoholism history, cancer, or transplantation; sarcoidosis; Hodgkin's lymphoma; collagen vascular disease; splenectomy; chronic organ failure; or 
systemic corticosteroid treatment (Cancelli et al., 2008). $\mathrm{CM}$, rare in immunocompetent patients whose treatment guidelines for these patients are based largely upon evidence from trials in HIV patients, although data suggests existence of significant differences in clinical presentation and prognosis between the two groups (Chau et al., 2010). It may affect people, regardless of age. However, the average age ranges from 30-40 years and men are more affected than women (Chau et al., 2010). Some studies show frequent confusion between Cryptococcosis neoformans e CM when initial symptoms are headache, fever followed by vomiting and seizures (Nakhayi et al., 2010).

The clinical course of $\mathrm{CM}$ is indolent with a median time to diagnosis from symptom onset of 44 days with a range of 7 days to 1 year. In patients with intact immune system, CM usually presents the typical signs and symptoms of meningitis: fever, stiff neck and headache. Visual changes have been reported, such as the field defects seen in our patient, as well as new onset seizure activity. Other ocular disorders include neuroretinitis, retinal hemorrhages, strabismus, nystagmus, anisocoria, ptosis, retinal exudates, optic nerve atrophy and ophthalmoplegia. Papilledema can be found in half of active cases, due to increased spinal fluid pressure. CM has also been shown to provoke ischemic brain injury. When the yeast spreads to the brain in immunocompetent hosts, the encapsulated fungus spurs a local granulomatous response and the fungus becomes "walled off," which may appear on Computed Tomography (CT) as a ring-enhancing lesion (Costa et al., 2009).

Complications are common, raised intracranial pressure in the absence of ventricular dilatation may cause profound visual or hearing loss. Less commonly, patients may develop cognitive impairment and gait ataxia due to obstructive hydrocephalus with ventricular dilatation. Much has been learnt about the immune response to cryptococcal infection from studies of animal models and from trials in vitro. In common with a number of other chronic fungal and bacterial infections, protection is associated with an active granulomatous inflammatory response and depends on intact cellmediated immunity involving both CD4 and CD8 cells and a Th1 pattern of cytokine release. Protective roles for Tumour Necrosis Factor- $\alpha$ (TNF- $\alpha)$, interleukins 12 and 18 (IL-12, IL-18) and Interferon- $\gamma$ (IFN- $\gamma$ ) have been inferred from experiments with knockout mice and antibody neutralization. Current studies have begun to define how C.neoformans stimulates an innate immune response through interaction with Toll-like receptors on host cell and the cryptococcal mannoproteins that are important in stimulating specific T-cell immunity. In addition, we have now directly correlated immune parameters at the site of infection in the Cerebrospinal Fluid (CSF) with survival and the rate of infection clearance determined by serial quantitative cultures of CSF over the first 2 weeks of therapy (Sachdeva et al., 2012).

Diagnostic evaluation for CM requires an LP with measurement of opening pressure; CSF analysis including cell counts, protein and glucose levels; India ink staining; fungal culture and cryptococcal antigen. Serum cryptococcal latex agglutination titers also should be drawn; these are $87 \%$ sensitive in HIV-negative patients. A definitive diagnosis of CM can be made by either visualizing the fungus in CSF using India ink preparation (75-85\% sensitive), CSF latex agglutination assay (95\% sensitive), or a positive CSF culture for $C$. neoformans. The CSF of patients with CM may be clear with increased lymphocytes and protein and decreased CSF glucose, or it may be turbid with increased cells, including polymorphonuclear leukocytes. Serum cryptococcal latex agglutination titers will be positive and WBC counts will be elevated in patients with intact immune systems (Chau et al., 2010).

Imaging tests may provide useful diagnostic information (although the findings are not pathognomonic and other infectious processes may still simulate cryptococcal infection in the brain). A communicating hydrocephalus may occur because of the acute meningeal exudate and also can occur late in the infection coursers because of meningeal adhesions. Gelatinous pseudocysts are seen as multiple CSFequivalent round or oval cysts in basal ganglia, thalami, midbrain, cerebellum and the periventricular regions. On MR imaging, these are seen as multiple hypointense T1 and hyperintense T2 lesions. Demonstration of clusters of these cysts in the basal ganglia and thalami is fairly specific and strongly suggestive of this infection (Charlier et al., 2008). The differential diagnosis for the malignant processes includes primary lung cancer with brain metastasis and other metastatic processes such as testicular cancer or melanoma, whereas the differential diagnosis for infectious processes includes bacteria (e.g., nocardia); mycobacteria; parasites (e.g., toxoplasma); and fungi such as cryptococcus, aspergillus, fusarium, zygomycetes, coccidioides, histoplasma and pseudoallescheria (Goldman et al., 2010).

As is true for other systemic mycoses, treatment of disease is due to $C$. neoformans having improved dramatically over the last 2 decades. Healthy hosts with CNS disease, standard therapy consists of Amphotericin 
B (AmB) deoxycholate $\left(\mathrm{AmBd} ; 0.7-1.0 \mathrm{mg} \mathrm{kg}^{-1}\right.$ per day Intravenously [IV]) plus flucytosine $\left(100 \mathrm{mg} \mathrm{kg}^{-1}\right.$ per day orally in 4 divided doses; IV formulations may be used in severe cases and in those without oral intake if the preparation is available) for at least 2 weeks, followed by fluconazole (400 $\mathrm{mg}\left[6 \mathrm{mg} \mathrm{kg}^{-1}\right]$ per day orally) for a minimum of 8 weeks (A-I). Lipid formulations of $\mathrm{AmB}$ (LFAmB), including liposomal AmB (3-4 mg kg-1 per day IV) and AmB lipid complex (ABLC; $5 \mathrm{mg} \mathrm{kg}^{-1}$ per day IV) for at least 2 weeks, could be substituted for AmBd among patients with or predisposed to renal dysfunction. In patient HIVnegative, AmBd (0.7-1.0 $\mathrm{mg} \mathrm{kg}^{-1}$ per day IV) plus flucytosine $\left(100 \mathrm{mg} \mathrm{kg}^{-1}\right.$ per day orally in 4 divided doses) for at least 4 weeks for induction therapy. The 4week induction therapy is reserved for people with meningoencephalitis without neurological complications and CSF yeast culture results that are negative after 2 weeks of treatment. For AmBd toxicity issues, LFAmB may be substituted in the second 2 weeks. In patients with neurological complications, consider extending induction therapy for a total of 6 weeks and LFAmB may be given for the last 4 weeks of the prolonged induction period. Then, start consolidation with fluconazole (400 mg per day) for 8 weeks (Perfect et al., 2010).

The addition of steroid therapy also helped to improve the patient's course, providing the medication to ameliorate this intense inflammatory response during the early stages of the infection. It is apparent that, by decreasing the junction between the endothelial cells, the steroids (which were tapered and then discontinued) prevented less permeability of the blood-brain barrier, decreasing passage of contrast material and the interstitial edema. A lumbar puncture is frequently recommended after 2 weeks of treatment to assess the status of CSF sterilization. But we find no evidence in the literature to support this commendation.

Problems with the use of amphotericin and flucytosine in our setting are due to the following: (a) costs of the drugs and prolonged hospitalization; (b) unavailability of the drugs; (c) unpredictable toxicity; (d) nonresponse to adequate treatment; and (e) relapse. There is a high mortality which can be attributed to one or more of following: (a) the disease itself; (b) complicating medical illness; (c) drug toxicity; (d) hydrocephalus (Sachdeva et al., 2012). Because of the low index of suspicion, the diagnosis is often delayed, resulting in poor prognosis with severe consequences. Neurological forms of cryptococcosis in immunocompetent patients needs to be considered in situations with intractable headache, papilloedema, hydrocephalus and prior to decisions on shunt placements (Costa et al., 2009). It is concluded that CM is uncommon but an important cause of non-acute meningitis and should be included in the list of causes of preventable blindness.

\section{CONCLUSION}

$\mathrm{CM}$ is rare in immunocompetent patients. Immunodeficiency primary, lymphoma or other clinical diseases that attend with an immunity decrease were investigated and discarded. This article aims advertising clinicians and specialists to the clinical manifestation and diagnosis of CM in immunocompetents, trying to avoid a late diagnosis or their complications.

\section{REFERENCES}

Baddley, J.W., J.R. Perfect, R.A. Oster, R.A. Larsen and G.A. Pankey et al., 2008. Pulmonary cryptococcosis in patients without HIV infection: Factors associated with disseminated disease. Eur. J. Clin. Microbiol. Infect. Dis., 27: 937-943. DOI: 10.1007/s10096-0080529-z

Cancelli, I., G. Merlino, A. Serafini, M. Valente and G.L. Gigli, 2008. Sarcoidosis as risk factor for cryptococcal meningitis in an apparently immunocompetent patient. Neurol. Sci., 29: 33-35. DOI: 10.1007/s10072-008-0856-y

Charlier, C., F. Dromer, C. Leveque, L. Chartier and Y.S. Cordoliani et al., 2008. Cryptococcal neuroradiological lesions correlate with severity during cryptococcal meningoencephalitis in HIVpositive patients in the HAART era. PLoS One, 3: e1950-e1950. DOI: 10.1371/journal.pone.0001950

Chau, T.T., N.H. Mai, N.H. Phu, H.D. Nghia and L.V. Chuong et al., 2010. A prospective descriptive study of cryptococcal meningitis in HIV uninfected patients in vietnam-high prevalence of Cryptococcus neoformans var grubii in the absence of underlying disease. Bmc. Infect. Dis., 10: 199-199. DOI: 10.1186/1471-2334-10-199

Chowdhary, A., H.S. Rhandhawa, A. Prakash and J.F. Meis, 2012. Environmental prevalence of Cryptococcus neoformans and Cryptococcus gattii in India: An update. Crit. Rev. Microbiol., 38: 1-16. DOI: 10.3109/1040841X.2011.606426 
Costa, M.L., J.P. Souza, A.F.O. Neto and J.L.P.E. Silva, 2009. Cryptococcal meningitis in HIV negative pregnant women: Case report and review of literature. Rev. Inst. Med. Trop. Sao. Paulo, 51: 289294. PMID: 19893983

Goldman, J.D., E.M. Vollmer and A.M. Luks, 2010. Cryptococcosis in the immunocompetent patient. Respiratory Care, 55: 1499-503.

Nakhayi, A.R., T.E. Eftekhaari, H. Montazerghaem, S. Moosavy and A. Madani, 2010. A 28 years old woman with severe headache and few episodes of vomiting: A case report. Am. J. Infect. Dis., 6: 107109. DOI: 10.3844 /ajidsp.2010.107.109
Perfect, J.R., W.E. Dismukes, F. Dromer, D.L. Goldman and J.R. Graybill et al., 2010. Clinical practice guidelines for the management of cryptococcal disease: 2010 update by the infectious diseases society of America. Clin. Infect. Dis., 50: 291-322. DOI: $10.1086 / 649858$

Sachdeva, R.K., S. Randev, A. Sharma, A. Wanchu and A. Chakrabarti et al., 2012. A Retrospective Study of AIDS-associated cryptomeningitis. AIDS Res. Hum. Retroviruses, 28: 1220-1226. DOI: 10.1089/aid.2011.0293 